\title{
Influence of steady shear flow on dynamic viscoelastic properties of un-reinforced and Kevlar, glass fibre reinforced LLDPE
}

\author{
TAKESHI KITANO ${ }^{\dagger}$, S A R HASHMI* and NAVIN CHAND \\ Regional Research Laboratory, Hoshangabad Road, Bhopal 462 026, India \\ ${ }^{\dagger}$ Research Centre of Macromolecular Technology, National Institute of Advanced Industrial Science and Technology \\ (AIST), 1-1-1, Higashi, Tsukuba, Ibaraki 305-0046, Japan
}

MS received 7 April 2004; revised 9 August 2004

\begin{abstract}
An experimental study was conducted to observe the effects of parallel-superposed flow condition on viscoelastic properties of LLDPE, Kevlar fibre reinforced LLDPE and hybrid of short glass fibre and Kevlar fibre reinforced LLDPE. Parallel-plate rheometer was employed for these tests. Rheological parameters such as loss modulus $\left(G^{\prime \prime}\right)$ and dynamic viscosity $\left(\eta^{\prime}\right)$ do not vary significantly on superposing steady state shear with oscillatory shear in the studied range of experiment at $185^{\circ} \mathrm{C}$ in un-reinforced LLDPE. Kevlar fibre reinforced LLDPE and Kevlar/glass fibre reinforced LLDPE showed significant changes in the flow behaviour under various sets of superposed conditions. Storage modulus $\left(G^{\prime}\right)$, and $G^{\prime \prime}$ become highly sensitive to low oscillatory angular frequencies $(\omega)$ under superposed conditions. These curves show two different regions with increased $\omega$ value. At low $\omega$ values, parameters $G^{\prime}$ and $G^{\prime \prime}$ change sharply reaching a certain value, thereafter, changes are moderate with increased $\omega$. In case of $\eta^{\prime}$ a maxima is observed, position of which, depends upon the value of steady shear rate. Maxima shifts towards higher frequencies with the increased steady shear rate.
\end{abstract}

Keywords. Kevlar fibre; glass fibre; LLDPE; rheological properties; hybrid composites.

\section{Introduction}

Flow properties of polymer (melts) are generally studied under steady state, or dynamic conditions. These studies provide important information useful to understand the viscoelastic behaviour of materials as well as provide required data to design a process of moulding, calendering, extrusion, stamping etc. The actual flow of polymers and their composites often involves highly time dependent strain histories and modes of deformation, which occur in plastic processing machines (Booij and Palmen 1982). The rheological behaviour of viscoelastic fluid undergoing parallel superposed steady and oscillatory shear flow offers the possibility to get information about structural parameters of nonlinear fluids (Osaki et al 1965; Masi et al 1982).

The filler filled polymer melts and fibre reinforced polymer melts show complex flow behaviour. The rheological properties of such heterogeneous system depend upon degree of flocculation of powder, interaction between dispersion medium and dispersed powder and the temporary network formed by the fibres, their entanglement etc. The structural density is also a function of volume fraction of reinforcing fibres (Amari et al 1992). The complex flow pattern encountered during moulding/ stamping are generally far from simple steady or oscilla-

\footnotetext{
*Author for correspondence
}

tory shear flow. Therefore, it is important to investigate the viscoelastic behaviour under the deformation outside the range of linear viscoelasticity by using superposition of oscillatory shear flow on steady shear flow. Isayev and Wong (1988), Wong and Isayev (1989) and Wong et al (1990) gave brief review of literature including theoretical and experimental studies related to parallel and orthogonal superposition of small and large amplitude oscillations upon steady shear flow. It was emphasized that dissipation of oscillatory energy is very important in parallel and orthogonal superposition of large amplitude oscillations on pressure flow for polymer melts in annular die. At higher frequencies heat conduction plays a vital role at low flow rates. It was suggested (McKenna and Zapas 1986) that superposition of small strains on large strains can be studied in terms of an incremental modulus which was observed to differ from small strain modulus, determined from experiments at zero pre-strain.

A large volume of polymer composites is being used in various applications having different compositions. Several high strength fibres such as Kevlar, carbon etc are frequently used in such high strength composites. Hybridized Kevlar and glass fibre reinforced LLDPE composites showed potential in tailoring the desired combination of mechanical properties (Hashmi et al 2003). The dynamic rheological studies of these composites have been reported (Kitano et al 2001; Hashmi et al 2002). The present work investigates the effect of parallel-superposed steady shear on dynamic viscoelastic properties of LLDPE, 
Kevlar fibre reinforced LLDPE and hybrid of Kevlar and glass fibres in LLDPE matrix.

\section{Materials and methods}

As reported earlier (Hashmi et al 2002), LLDPE powder (type-A $220 \mathrm{~J}$, having density, $0.923 \mathrm{~g} / \mathrm{cc}$, and M.F.I., $20 \mathrm{~g} / 10 \mathrm{~min}$ ) was obtained from Japan Poly Olefins Co. Ltd., Japan and was used as matrix material. Reinforcing fibres for hybrid composite preparation were glass fibres and Kevlar fibres. Glass fibres having density, $2.57 \mathrm{~g} / \mathrm{cc}$, initial length, $5 \mathrm{~mm}$ diameter, $13 \mu \mathrm{m}$, was supplied by Nihon Glass Fibre, Japan and Kevlar fibres having density, $1.48 \mathrm{~g} / \mathrm{cc}$, length, $10 \mathrm{~mm}$, diameter, $12 \mu \mathrm{m}$, was supplied by Toray - Dupont, Japan.

One set of 20 vol.\% of fibres was reinforced in LLDPE matrix. The composition of Kevlar and glass fibres was varied as $25,50,75$ and $100 \mathrm{vol} . \%$ of total volume of fibres in composite. The samples were designated as per following scheme:

Volume\% of Kevlar fibre - volume\% of glass fibre/ volume of LLDPE :: KF-GF/LL. Total volume of fibres was $20 \%$ in LLDPE. In this $20 \mathrm{vol} \%$ of fibres the ratio of Kevlar and glass fibres was varied.

LLDPE powder was dry blended with fibres and then extruded through an elastic melt extruder. The elastic melt extruder works on the Weissenberg principle (Kataoka et al 1976). The material was fed directly into the shearing zone, which was built by the rotor and stator. The effective rotor diameter was $14 \mathrm{~cm}$. The temperature of extruder was kept constant at $200^{\circ} \mathrm{C}$ for all compositions. The extruded material was quenched in water at $12^{\circ} \mathrm{C}$ and was immediately cut by pelletizer to a $5 \mathrm{~mm}$ length. The pellets were dried in an air-circulating oven at $75^{\circ} \mathrm{C}$ for $12 \mathrm{~h}$. The above process was repeated once to achieve better mixing. A weighed amount of pellets was placed in the mould cavity. The material was compressed between the hot plates for $3 \mathrm{~min}$ under $5 \mathrm{MPa}$ pressure at $200^{\circ} \mathrm{C}$. The mould was then cooled immediately under the same pressure by circulating water at $12^{\circ} \mathrm{C}$ and sheets of $2.5-$ $3 \mathrm{~mm}$ thickness were prepared. These sheets were cut to disc shape for tests by using a sawing machine. These discs were tested on a parallel plate type rheometer, NRM2000, from Nippon Rheology Ki-Ki Co. Ltd. Plate radius was $R=2 \cdot 15 \mathrm{~cm}$. Tests were conducted at different angular frequencies that were varied from 0.031 to $37.7 \mathrm{rad} / \mathrm{s}$. All the measurements were taken at $185^{\circ} \mathrm{C}$. Strain amplitude was kept constant at $10 \%$. Steady shear rate applied on oscillatory flow of samples was varied in five steps as $0 \cdot 01,0 \cdot 05,0 \cdot 1,0 \cdot 5$ and $1 \cdot 0 \mathrm{~s}^{-1}$.

\section{Results and discussion}

The total stress, $\tau_{t}$, aroused from a combined motion is composed of a stationary contribution of steady shear, $\tau$, and a fluctuating part due to oscillatory motion, $\tau_{0}$ and can be written in the following form (Pipkin and Owen 1967)

$$
\tau_{\mathrm{t}}=\eta \gamma^{\prime}+\left(\eta^{\prime} \gamma_{0}^{\prime} / \sin \delta\right) \sin (\omega t+\delta),
$$

where $\eta$ is the fluid viscosity that may be shear rate, $\gamma^{\prime}$, dependent or independent, $\eta^{\prime}$ the dynamic viscosity, $\gamma_{0}^{\prime}$ the amplitude of shear wave and $\delta$ the phase lag between stress and deformation.

Other important relation used in this study is obtained from literature (Pipkin 1968; Powel and Schwarz 1979) for superposed parallel flow in parallel plate geometry of rheometer.

$$
\lim _{\omega \rightarrow 0} \eta^{\prime \prime} / \omega=\lim _{\omega \rightarrow 0} G^{\prime} / \omega^{2}=\text { constant }
$$

where $G^{\prime}$ is storage modulus and $G^{\prime}=\omega \eta^{\prime \prime}$.

Figure 1 shows a plot between storage modulus $\left(G^{\prime}\right)$ and angular frequency $(\omega)$ for various samples of KF-GF/LL composites of varying composition under parallel superposed flow. The steady state shear rate was fixed at $0.1 \mathrm{~s}^{-1}$ and angular frequency was varied from $0.03-37.70 \mathrm{rad} / \mathrm{s}$. It is evident from figure 1 that $G^{\prime}$ increases with $\omega$ while keeping the strain amplitude constant in all cases. Initially, at low frequencies, $G^{\prime}$ increases sharply with $\omega$ however, after reaching a certain frequency, the effect of variation of $\omega$ on $G^{\prime}$ reduces significantly. The effect of composition is significant in this range of experiments.

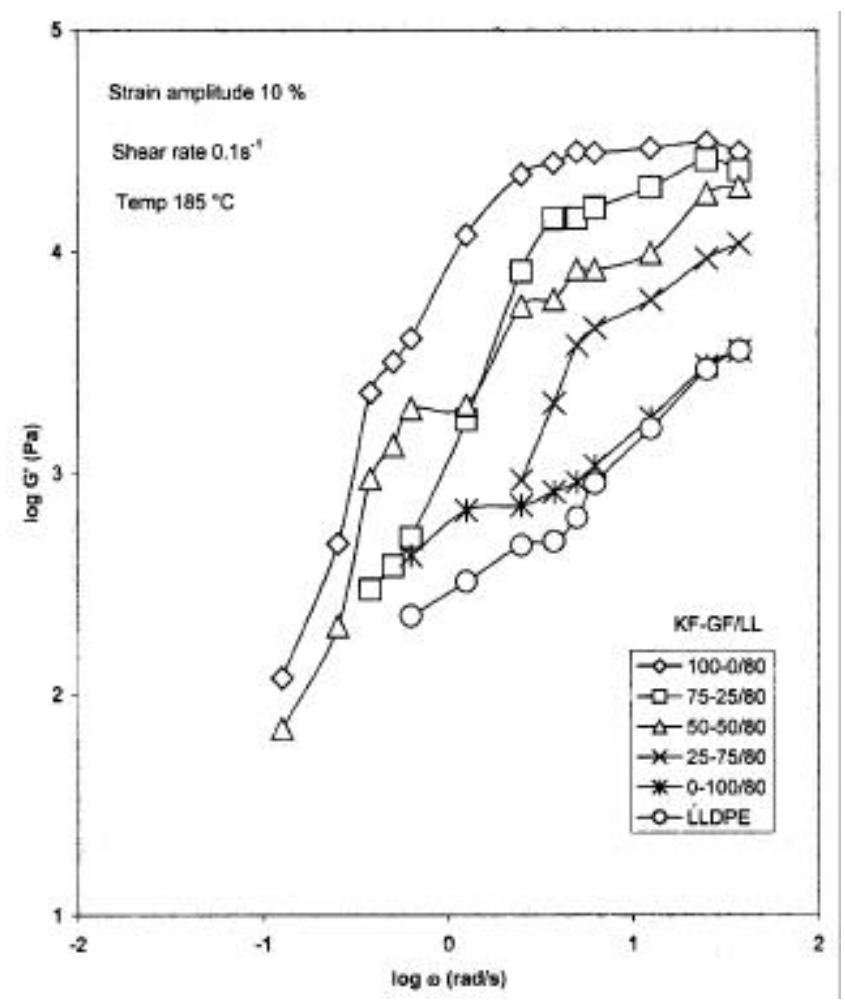

Figure 1. $G^{\prime}$ as a function of $\omega$ for different compositions of $\mathrm{KF}-\mathrm{GF} / \mathrm{LL}$ composites at fixed steady shear rate. 
LLDPE shows almost linear relationship between logarithmic values of $G^{\prime}$ and $\omega$. The reinforcement of LLDPE with either Kevlar fibre or glass fibre significantly affects the slope of the curve that increases with reinforcement. Moreover, slope of the curve belonging to Kevlar fibre reinforced LLDPE is steeper than that of glass fibre reinforced LLDPE. This effect reflects on the values of $G^{\prime}$ of intermediate compositions. The replacement of glass fibre with Kevlar fibre increases the value of $G^{\prime}$. This is attributed to the higher modulus of Kevlar that is added to the modulus of molten LLDPE and the larger length of Kevlar fibres as compared to glass fibres. It has been previously shown that the process of mixing the ingredients severely damage and reduces the length of glass fibre as compared to Kevlar fibre. The length of Kevlar fibre does not change much during mixing process. This behaviour has been reported earlier in this KF-GF/LL system (Hashmi et al 2003) under dynamic conditions. Similar effects were observed here under superposed flow conditions.

Figure 2 shows a plot between loss modulus $\left(G^{\prime \prime}\right)$ and angular frequency for various compositions of KF-GF/LL composite. $G^{\prime \prime}$ increases with angular frequency. The value of $G^{\prime \prime}$ increases with reinforcement. Addition of Kevlar changes $G^{\prime \prime}$ more significantly than glass fibre. The replacement of glass fibre with Kevlar fibre increases the value of $G^{\prime \prime}$.

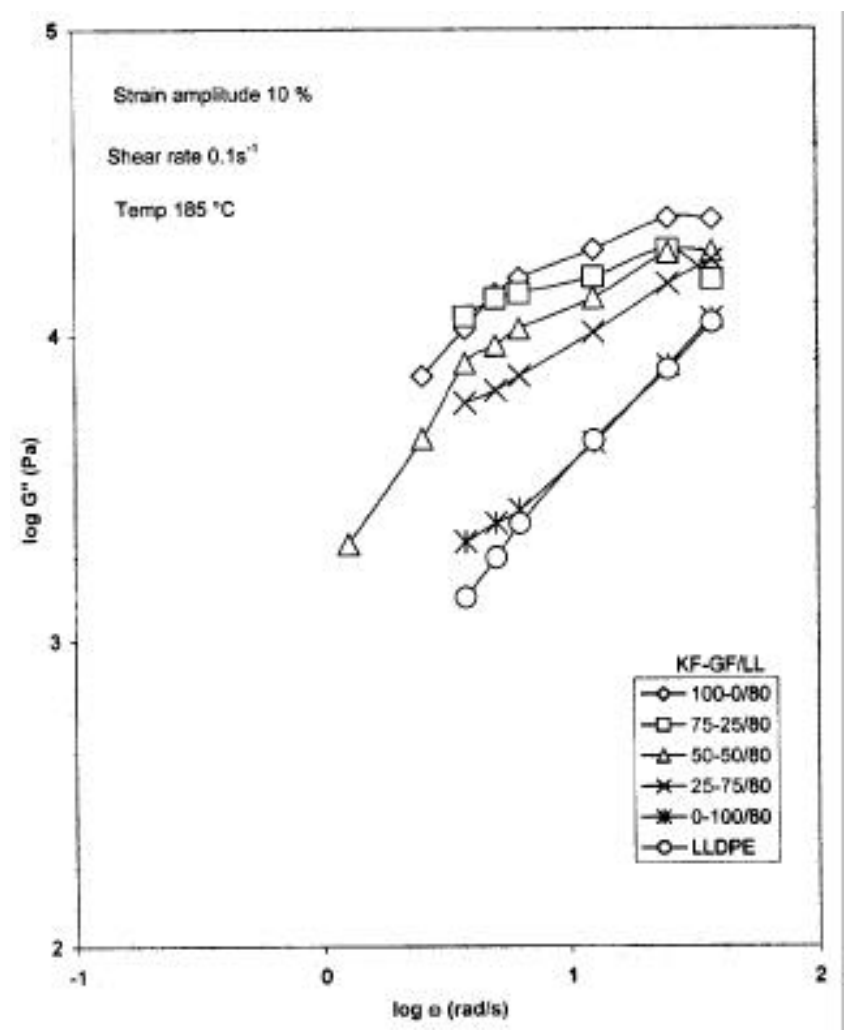

Figure 2. $G^{\prime \prime}$ as a function of $\omega$ for different compositions of KFG-GF/LL composites at fixed steady shear rate.
Figure 3 shows a plot between dynamic viscosity, $\eta^{\prime}$, and angular frequency for various samples of $\mathrm{KF}-\mathrm{GF} / \mathrm{LL}$ composites of varying composition. The value of dynamic viscosity decreases with angular frequency and increases with reinforcement. Addition of Kevlar changes $\eta^{\prime}$ more significantly than glass fibre. The replacement of glass fibre with Kevlar fibre increases the value of $\eta^{\prime}$. LLDPE does not show the sensitivity with angular frequency and behaves as Newtonian fluid at $185^{\circ} \mathrm{C}$. The addition of fibres changed the system to non-Newtonian. Shear thinning effect was observed in all the systems.

Figure 4 is a plot between logarithmic values of $G^{\prime}$ and $\omega$ for composite $\mathrm{KF}-\mathrm{GF} / \mathrm{LL}:: 75-25 / 80$ under parallel superposed flow conditions. The various curves are drawn with respect to varied steady state shear rate at the fixed strain amplitude of $10 \%$. The value of $G^{\prime}$ appears to be less affected with $\omega$ under only oscillatory conditions as compared to those curves belonging to superposed flow conditions. At low angular frequencies the values of $G^{\prime}$ is more sensitive to $\omega$ values. On introducing the steady state shear with oscillatory shear rate, it was observed that $G^{\prime}$ becomes highly sensitive to $\omega$ and increases with $\omega$ at the low $\omega$ values. The sensitive region of $\omega$ shifted to higher $\omega$ values with the increased steady shear rate $(\gamma)$ values. All the curves belonging to superposed flow are nearly parallel to each other at higher angular frequencies, how-

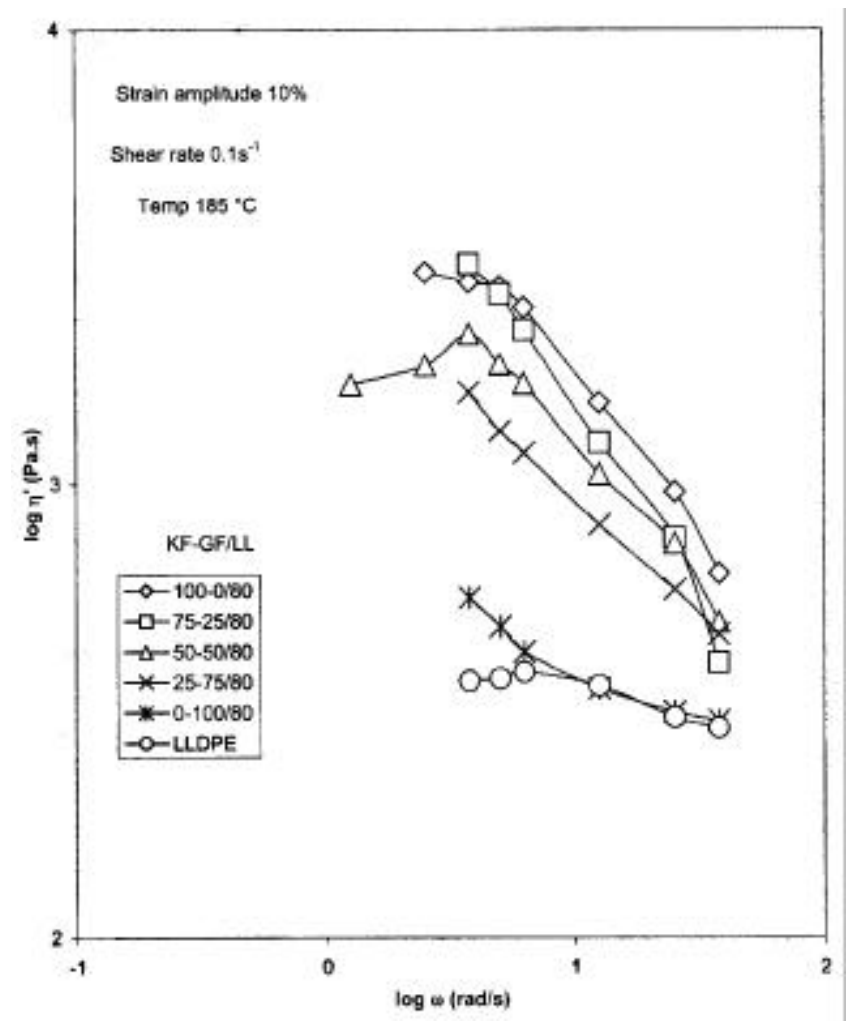

Figure 3. $\eta^{\prime}$ as a function of $\omega$ for different compositions of $\mathrm{KF}-\mathrm{GF} / \mathrm{LL}$ composites at fixed steady shear rate. 
ever, the values of $G^{\prime}$ reduced with increased shear rate values. Reduction in $G^{\prime}$ under superposed steady shear flow of non-Newtonian liquids increases with increased $\gamma$ values. This is attributed to reduced elasticity of material under superposed flow conditions in which the large deformation leads to plastic flow of material.

Figure 5 is a plot between logarithmic values of $G^{\prime \prime}$ and $\omega$ for composite KF-GF/LL :: 75-25/80 under parallel superposed flow conditions. The various curves are drawn with respect to varied steady state shear rate at fixed strain amplitude of $10 \%$. The value of $G^{\prime \prime}$ appears to be less affected with $\omega$ under oscillatory conditions as shown in figure 5 as compared to those curves belonging to superposed flow conditions. At low angular frequencies the values of $G^{\prime \prime}$ is more sensitive to $\omega$ values. On introducing the steady state shear with oscillatory shear rate, it was observed that $G^{\prime \prime}$ becomes highly sensitive to $\omega$ and increases with $\omega$ at low $\omega$ values. The sensitive region of $\omega$ shifted to higher $\omega$ values with the increased $\gamma$ values. All the curves belonging to superposed flow are nearly parallel to each other at higher frequencies, however, the values of $G^{\prime \prime}$ reduces with increased $\gamma$ values. This is attributed to shear thinning of material under superposed flow conditions.

Figure 6 is a plot between logarithmic values of $\eta^{\prime}$ and $\omega$ for composite $\mathrm{KF}-\mathrm{GF} / \mathrm{LL}:: 75-25 / 80$ under parallel superposed flow conditions. The dynamic viscosity, $\eta^{\prime}$,

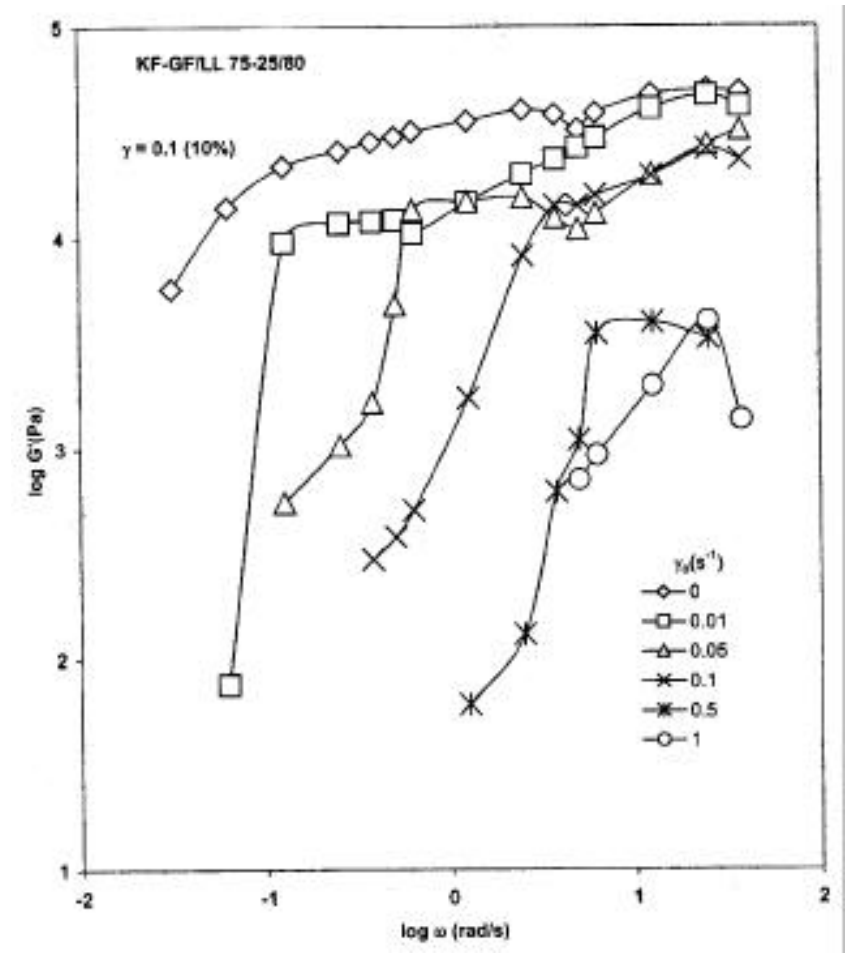

Figure 4. $G^{\prime}$ as a function of $\omega$ for hybrid composition of $\mathrm{KF}-\mathrm{GF} / \mathrm{LL}::$ 75-25/80 composites at different superposed steady shear rates.

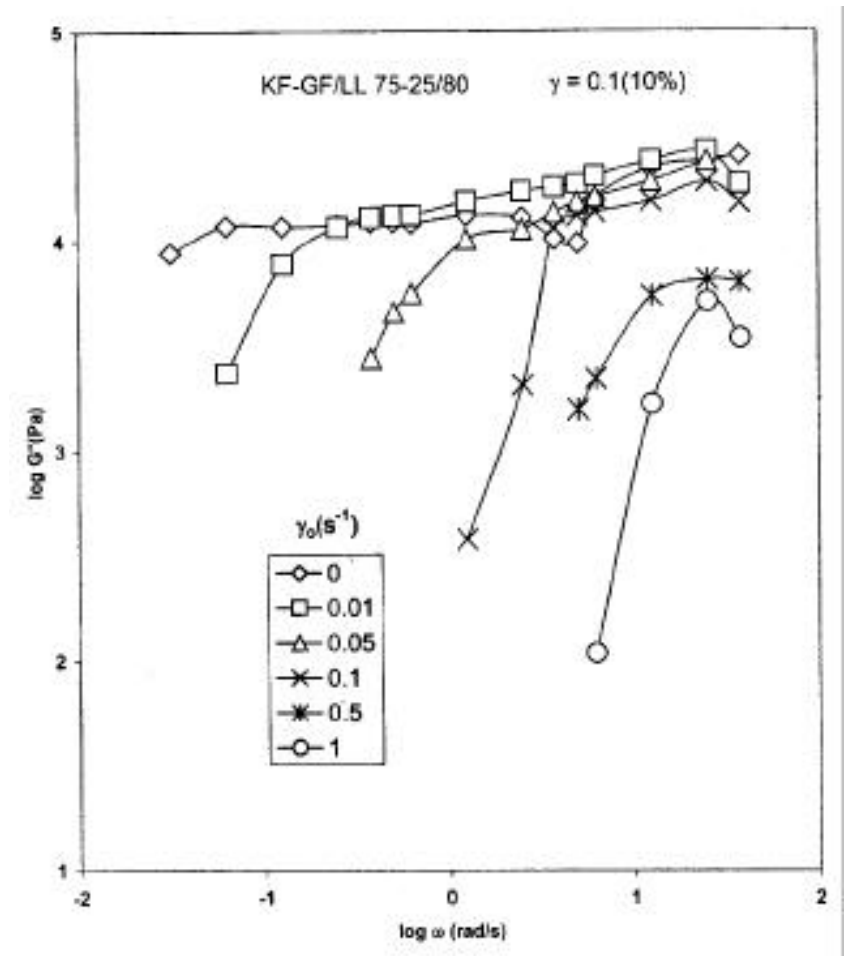

Figure 5. $G^{\prime \prime}$ as a function of $\omega$ for hybrid composition of $\mathrm{KF}-\mathrm{GF} / \mathrm{LL}::$ 75-25/80 composites at different superposed shear rates.

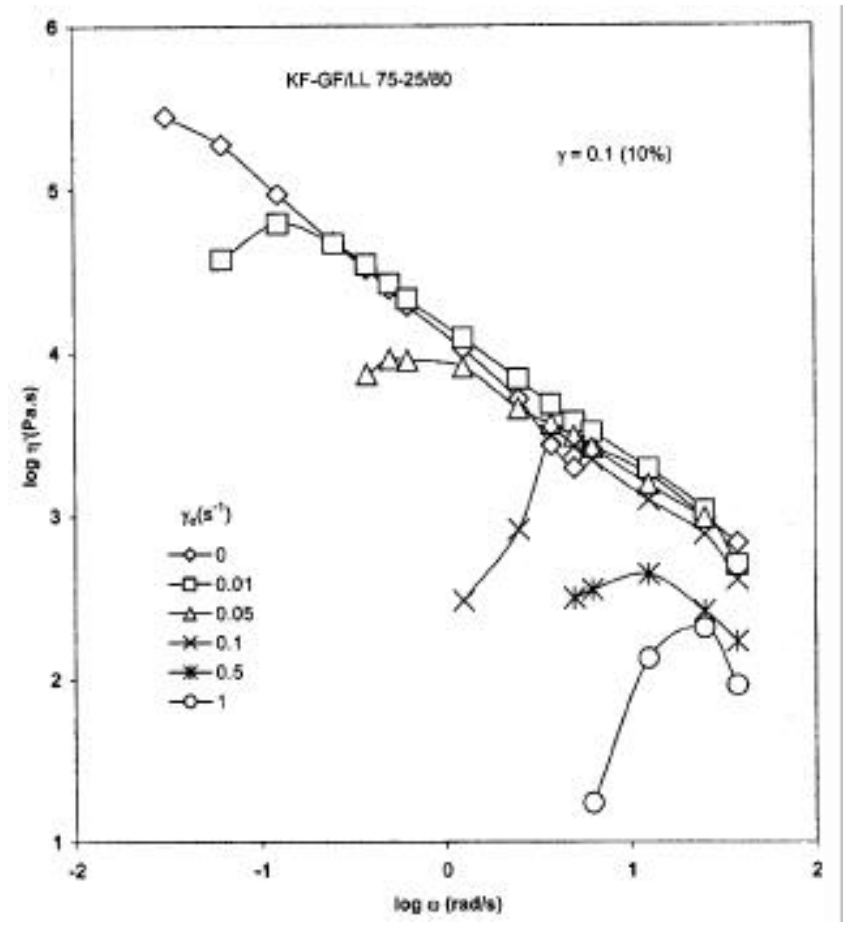

Figure 6. $\eta^{\prime}$ as a function of $\omega$ for hybrid composition of KFGF/LL :: 76-26/80 composite at different superposed steady shear rates. 
decreases with $\omega$ in the whole range of experiment, showing shear thinning effect under oscillatory shear conditions. On introducing the steady state shear with oscillatory shear, it was observed that $\eta^{\prime}$ shows a maxima in $\eta^{\prime}$ vs $\omega$ curve. The dynamic viscosity increases with $\omega$ at low angular frequencies, reaches a maximum value and then reduces with increased $\omega$. The region in which maxima falls shifted to higher $\omega$ values with the increased $\gamma$ values.

Figures 7-9 are the plots between $\log G^{\prime}, \log G^{\prime \prime}, \log$ $\eta^{\prime}$ vs $\log \omega$, respectively for un-reinforced LLDPE under simple oscillatory and superposed flow conditions. As compared to the curves belonging to reinforced LLDPE, the significant difference is obvious. Reinforced LLDPE shows significant changes on superposing steady shear on dynamic shear whereas un-reinforced LLDPE does not appear to be significantly affected on $G^{\prime}, G^{\prime \prime}$ and $\eta^{\prime}$ by superposing shear. The reinforcement strongly affects the rheological properties under superposed flow conditions.

Figures 10-12 are plotted for $\log G^{\prime}, \log G^{\prime \prime}$ and $\log$ $\eta^{\prime}$ vs $\log \omega$, respectively for Kevlar fibre reinforced LLDPE composites. These plots are similar to those shown in figures 4-6 where hybrid of glass, Kevlar-fibre reinforced LLDPE material was studied. The values of $G^{\prime}, G^{\prime \prime}$ and $\eta^{\prime}$ are comparatively higher in Kevlar fibre reinforced composites as compared to hybrid fibre reinforced LLDPE. This is attributed to replacing glass fibre

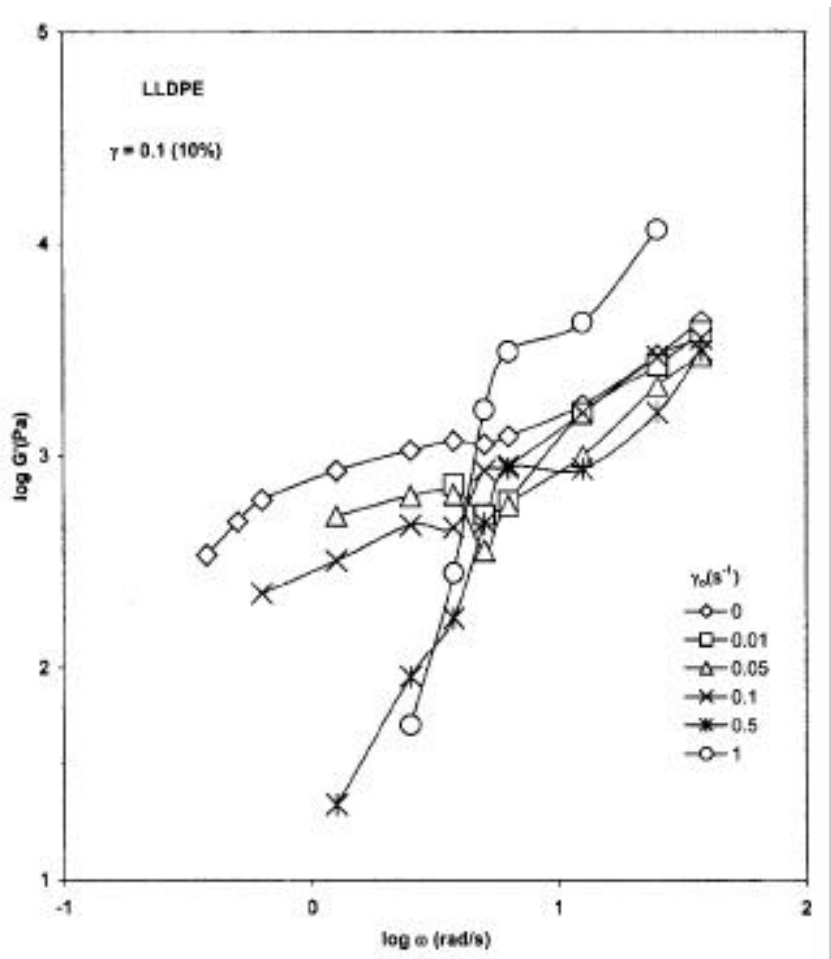

Figure 7. $G^{\prime}$ as a function of $\omega$ for LLDPE at different superposed steady shear rates. by longer and higher modulus Kevlar fibres in LLDPE, as the volume fraction of fibres in both sets is almost same.

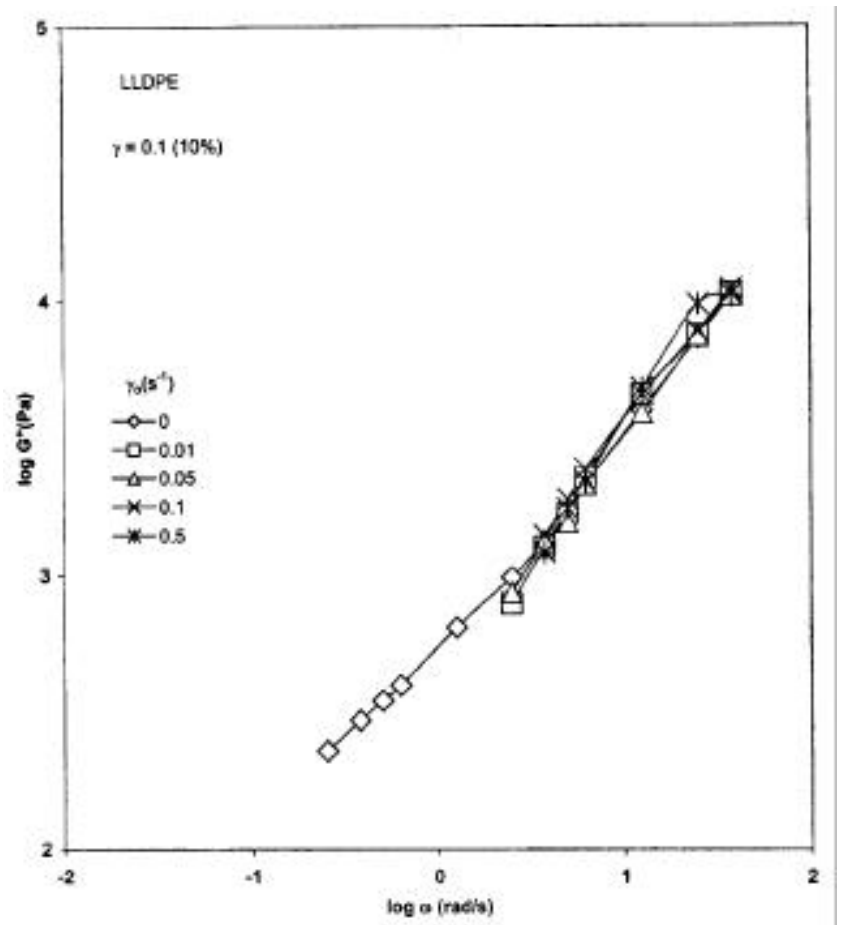

Figure 8. $G^{\prime \prime}$ as a function of $\omega$ for LLDPE at different superposed steady shear rates.

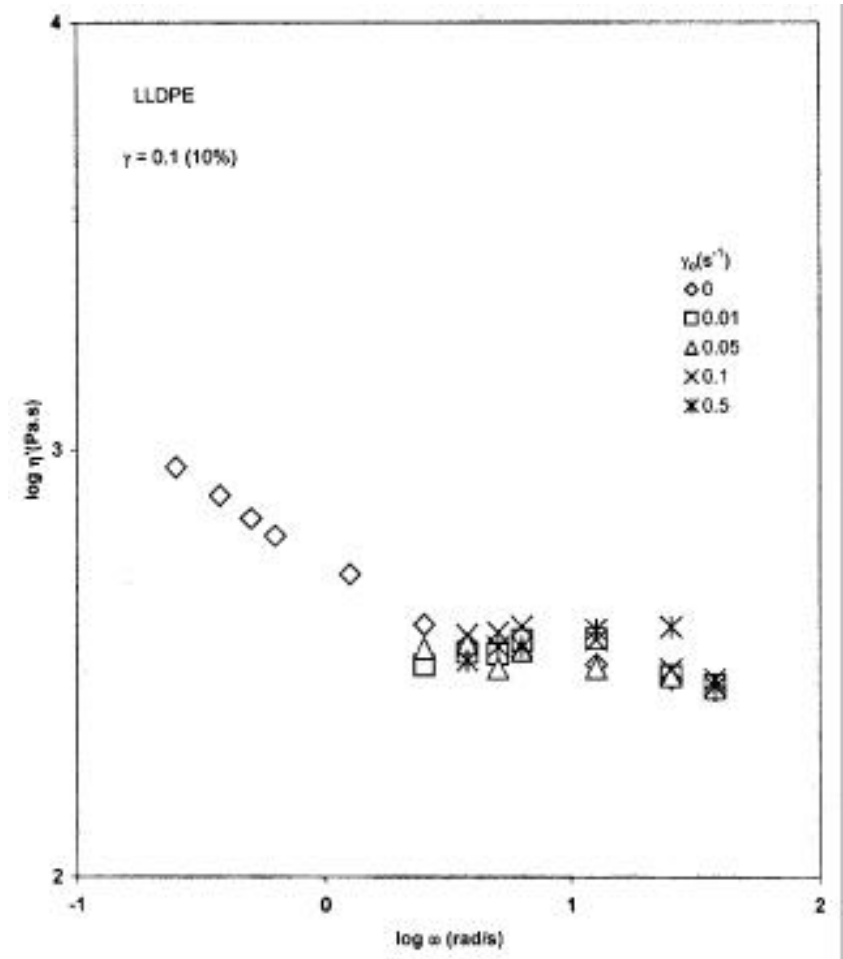

Figure 9. $\eta^{\prime}$ as a function of $\omega$ for LLDPE at different superposed steady shear rates. 


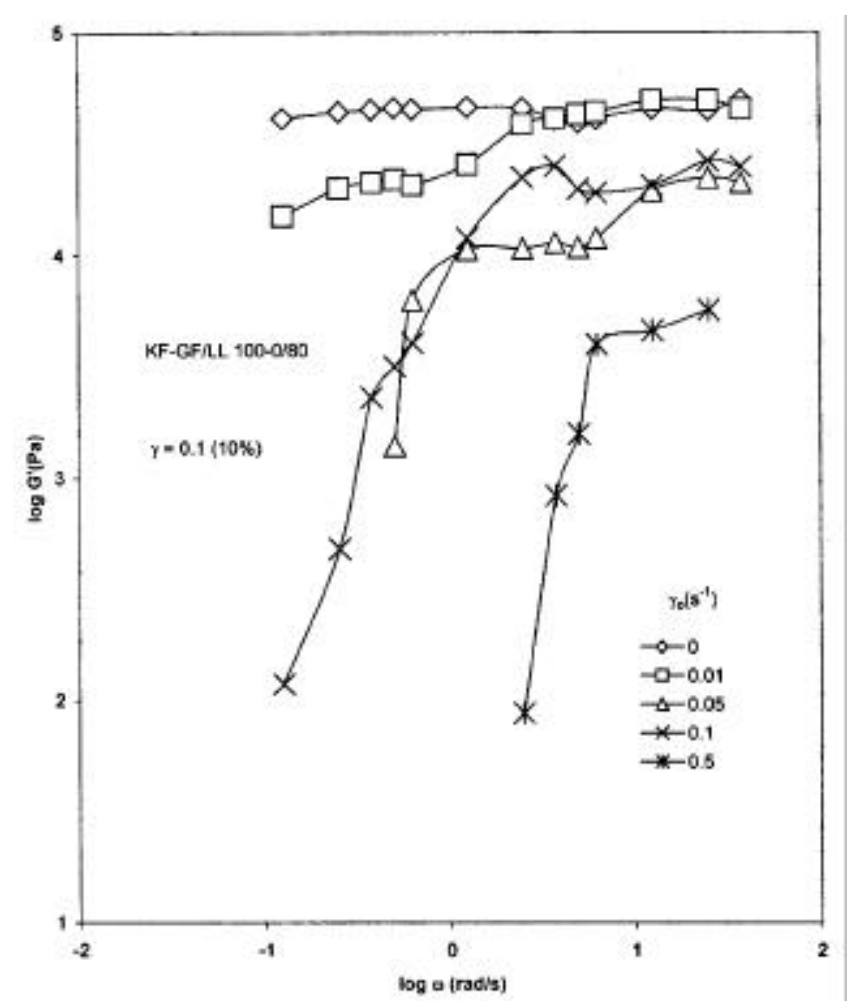

Figure 10. $G^{\prime}$ as a function of $\omega$ for Kevlar fibre reinforced LLDPE (KF-GF/LL :: 100-0/80) at different superposed steady shear rates.

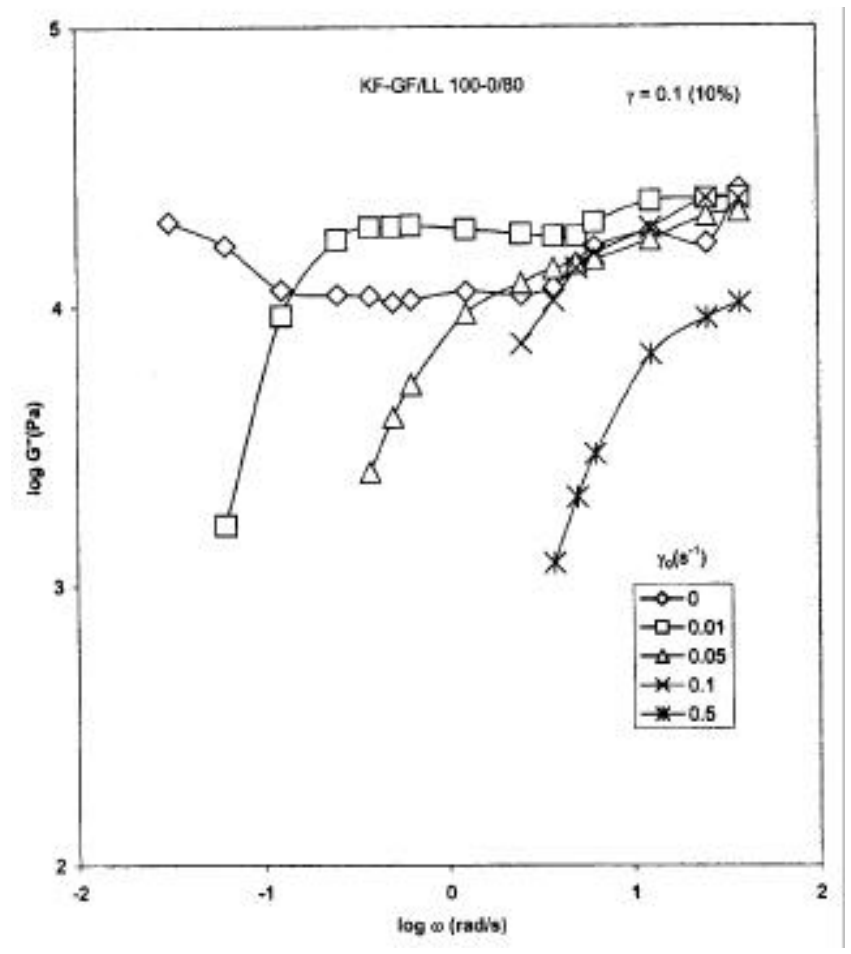

Figure 11. $G^{\prime \prime}$ as a function of $\omega$ for Kevlar fibre reinforced LLDPER (KF-GF/LL :: 100-0/80) at different superposed steady shear rates.

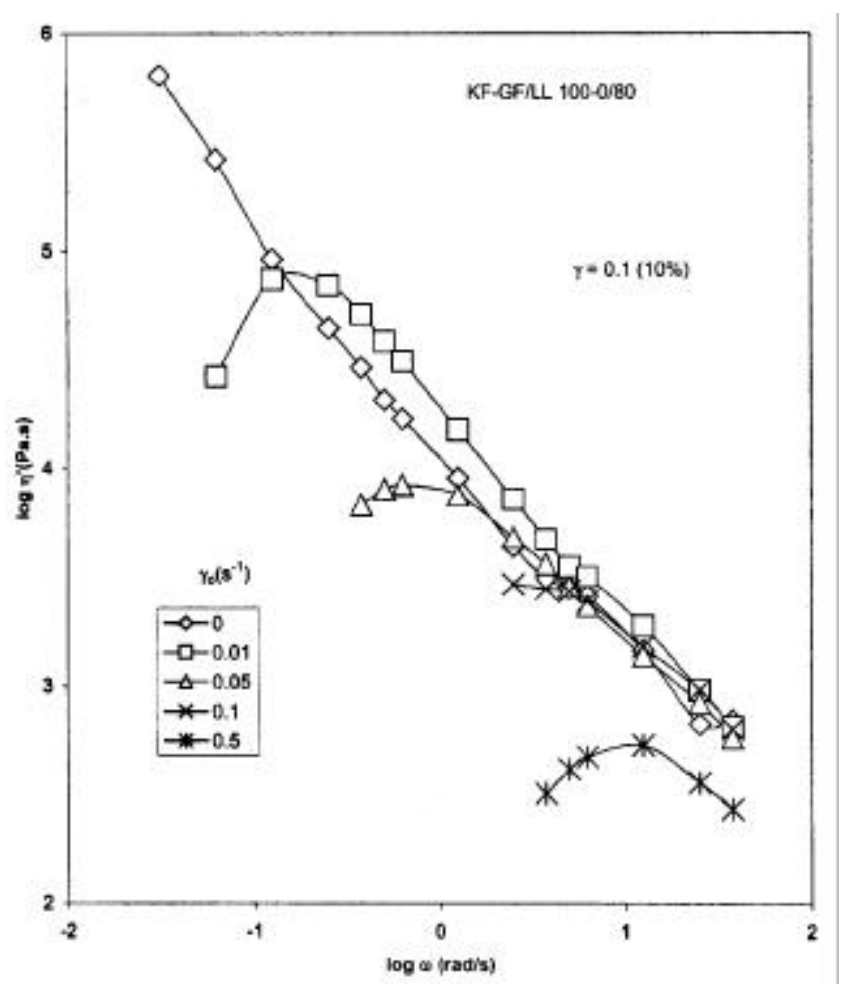

Figure 12. $\eta^{\prime}$ as a function of $\omega$ for Kevlar fibre reinforced LLDPE (KF-GF/LL :: 100-0/80) at different superposed steady shear rates.

\section{Conclusions}

Experimental study on parallel-superposed flow condition on the dynamic viscoelastic properties of LLDPE, Kevlar fibre reinforced LLDPE and hybrid of short glass fibre and Kevlar fibre reinforced LLDPE was conducted and the following effects were observed:

(I) $G^{\prime \prime}$ and $\eta^{\prime}$ do not vary significantly on superposing steady state shear with oscillatory shear in the studied range of experiment at $185^{\circ} \mathrm{C}$ in un-reinforced LLDPE.

(II) Kevlar fibre reinforced LLDPE shows significant changes in the flow behaviour under various sets of superposed conditions. The superposed conditions produce maximum effects at low oscillatory angular frequencies where $G^{\prime}, G^{\prime \prime}$ and $\eta^{\prime}$ become highly sensitive to $\omega$. These curves show two different regions with increased $\omega$ value. At low $\omega$ values, parameters $G^{\prime}, G^{\prime \prime}$ and $\eta^{\prime}$ change sharply reaching a certain value, thereafter changes are moderate with increased $\omega$. In case of $\eta^{\prime}$, a maxima is observed, and the position of which depends upon steady shear rate. Maxima shifts towards higher angular frequencies with the increased steady shear rate. (III) Kevlar and glass fibre reinforced LLDPE hybrid composite shows similar behaviour under superposed 
conditions as mentioned above for Kevlar fibre reinforced LLDPE.

(IV) Slope of curve between $G^{\prime}$ and $\omega$ increases with reinforcement. The slope of glass fibre reinforced LLDPE was less steep than that of Kevlar fibre reinforced LLDPE.

\section{References}

Amari T, Wei X and Watanabe K 1992 Theoretical and applied rheology, Proc. XIth Int. Congress on Rheology (eds) $\mathrm{P}$ Moldenaers and R Keunings (Brussels: Elsevier Science Publisher) p. 573

Booij H C and Palmen J H M 1982 Rheol. Acta 21376

Hashmi S A R, Kitano T and Chand N 2002 Polym. Compos. 23500

Hashmi S A R, Kitano T and Chand N 2003 Polym. Compos. 24149
Isayev A I and Wong C M 1988 J. Polym. Sci. Polym. Phys. Ed. 262303

Kataoka T, Ohnishi S, Kitano T, Nakama K and Takayama H 1976 Rheol. Acta 15268

Kitano T, Hashmi S A R and Chand N 2001 Appl. Rheol. 11 258

Masi P, Nicodemo L, Nicolais L and Taglialatela G 1982 Rheol. Acta 21598

McKenna G B and Zapas L J 1986 Polym. Eng. Sci. 26 725

Osaki K, Tamura M, Kurata M and Kotaka T 1965 J. Phys. Chem. 694185

Pipkin A C 1968 Trans. Soc. Rheol. 12397

Pipkin A C and Owen D R 1967 Phys. Fluids 10836

Powel R L and Schwarz W H 1979 J. Polym. Sci. Polym. Phys. Ed. 17969

Wong C M and Isayev A I 1989 Rheol. Acta 28176

Wong C M, Chen C H and Isayev A I 1990 Polym. Eng. Sci. 30 1574 